Article

\title{
Effect of different types of warm-up on strength and skin tem- perature of Paralympic powerlifting athletes
}

\author{
Marcelo de Aquino Resende1, Felipe J. Aidar ${ }^{2,3,4,5}$, Roberta Barreto Vasconcelos Resende ${ }^{1}$, Gracielle Costa Reis ${ }^{1}$, \\ Layanne de Oliveira Barros², Dihogo Gama de Matos ${ }^{2,6}$, Anderson Carlos Marçal ${ }^{2,3}$, Paulo Francisco de Almeida- \\ Neto $^{7}$, Alfonso López Díaz-de-Durana ${ }^{8}$, María Merino Fernández ${ }^{9}$, José Vilaça-Alves ${ }^{10}$, Breno Guilherme de Araújo \\ Tinoco Cabral7, Eduardo Borba Neves ${ }^{11}{ }^{*}$, Victor Machado Reis ${ }^{10}$, Nuno Domingos Garrido ${ }^{10}$
}

1. Department of Physical Education, Tiradentes University (UNIT), Aracaju, Sergipe, 49010-390, Brazil.

2. Group of Studies and Research of Performance, Sport, Health and Paralympic Sports (GEPEPS), Federal University of Sergipe (UFS), São Cristovão, Sergipe, 49100-000, Brazil.

3. Program of Physical Education, Federal University of Sergipe (UFS), São Cristovão, Sergipe, 49100000, Brazil.

4. Program of Physiological Science, Federal University of Sergipe (UFS), São Cristovão, Sergipe, 49100-000, Brazil.

5. Department of Physical Education, Federal University of Sergipe (UFS), São Cristovão, Sergipe, 49100-000, Brazil.

6. Cardiovascular \& Physiology of Exercise Laboratory, University of Manitoba, Winnipeg, R3T 2N2, Canada

7. Department of Physical Education, Federal University of Rio Grande do Norte (UFRN), Natal, Rio Grande do Norte, 59078-970, Brazil

8. Sports Department. Physical Activity and Sports Faculty-INEF, Universidad Politécnica de Madrid, Madrid, Spain

9. Faculty of Health Sciences. Universidad Francisco de Vitoria (UFV), Madrid, Spain

10. Research Center in Sports Sciences, Health Sciences and Human Development (CIDESD), Trás os Montes and Alto Douro University, 5001-801 Vila Real, Portugal

11. Universidade Tecnológica Federal do Paraná (UTFPR), 80230-901 Curitiba-PR, Brazil

Citation: Lastname, F.; Lastname, F.; Lastname, F. Title. Life 2021, 11, x. https://doi.org/10.3390/xxxxx

* Correspondence: educaroneves@utfpr.edu.br; Tel.: +55-2199530-4321 (E.B.N)

\begin{abstract}
Background: The aim of the present study was to evaluate the effect of different types of warm-ups on the strength and skin temperature of Paralympic powerlifting athletes; (2) Methods: The participants were 15 male Paralympic powerlifting athletes. It was analyzed the effects of three different types of warm-up (without warm-up (WW), traditional warm-up (TW), or stretching warm-up (SW)) on static and dynamic strength tests as well as in the skin temperature, which was monitored by thermal imaging; (3) Results: show no differences in the dynamic and static indicators of the force in relation to the different types of warm-up. No significant differences were found in relation to the Peak Torque ( $\mathrm{p}=0.055, \mathrm{~F}=4.560, \eta 2 \mathrm{p}=0.246$ medium effect), and 1-Repetition Maximum $(p=0.139, F=3.191, \eta 2 p=0.186$, medium effect) between the different types of warm-up. In the thermographic analysis, there was a significant difference only in the Pectoral muscle clavicular portion between the TW $\left(33.04 \pm 0.71^{\circ} \mathrm{C}\right)$ and the WW $\left(32.51 \pm 0.74^{\circ} \mathrm{C}\right)(\mathrm{p}=0.038)$. The TW method also presented slightly higher values than the SW and WW in the Pectoral Muscles Sternal portion and in the Deltoid anterior portion, but with p-value $>0.05$; (4) Conclusions: that the types of warmup studied do not seem to interfere with the performance of Paralympic Powerlifting athletes. However, the thermal images showed that traditional warm-up best meets the objectives expected for this preparation phase.
\end{abstract}

Keywords: warm up, muscle force, performance, resistance training, thermal imaging, physiology 


\section{Introduction}

Physical activity has been described as an important way to promote physical and psychosocial health $[1,2,3]$, in special groups $[4,5,6,7,8,9,10,11]$ and disabled people [12]. Among physical activities, strength training has gained greater attention with several studies indicating better physical and psychosocial indicators $[13,14,15]$. On the other hand, strength training with the intention of competition has been promoted with disabled people and with improvements in physical parameters [12,16].

Among the strength sports, the Paralympic Powerlifting (PP) has been gaining more and more followers, and the sport aims to lift the greatest possible load in the adapted bench press $[16,17,18]$. However, the studies have focused more on aspects of health, classification and etiology of injuries $[12,19,20]$. On the other hand, few studies have addressed aspects related to performance $[18,21,22,23]$.

When addressing the issue of performance in adapted sports and the Powerlifting Paralympic, the warm-up is presented as essential for performance [24]. Warming aims at improving nerve conduction, allied to an increase in temperature $[25,26]$. The specific warm-up has been shown to improve strength $[27,28,29]$, however, variations in the type of warm-up can be harmful [30]. Currently, there is no consensus between the effect of different types of warm-ups $[29,31,32]$.

Therefore, the aim of the present study was to evaluate the effect of different types of warm-ups on the strength and skin temperature of Paralympic powerlifting athletes. It was hypothesized that the warm-up methods are not capable of altering the performance of paralympic powerlifting athletes.

\section{Materials and Methods}

\subsection{Sample}

Fifteen male Paralympic powerlifters volunteered for this study. Every participant was a competitor involved in national competitions and were eligible for this sport under the International Paralympic Committee. Participants were required to have participated in a minimum of one competition at the national level over the past 12-month period and average prior experience in the sport was $2.43 \pm 1.03$ years. The participants mean age and body mass was $28.47 \pm 5.79$ years and $81.75 \pm 17.33 \mathrm{~kg}$, respectively. Body mass was assessed with specific adapted equipment as described Resende et al., [32].

\subsection{Experimental Design}

The study comprised 3 weeks which included 9 sessions separated by a minimum of 48-h. The first three sessions (week 1 ) were dedicated to baseline measurements of thermal images on session 1, and to familiarization with the dynamic strength tests on sessions 2 (1-RM and mean propulsive velocity) and the isometric strength tests on session 3 (impulse, variability, peak torque). In week 2 (sessions 4 to 6 ), the subjects performed in random order the three experimental conditions herein (3 types of warm-up) followed 10min later by the dynamic strength tests [33]. Skin temperature was measured immediately post-warm-up. In week 3 (sessions 7 to 9), the subjects performed in random order the three experimental conditions herein (3 types of warm-up) followed 10-min later by the isometric strength tests.

All testing was performed in an acclimated room, at the same time of day for and under the same environmental conditions $\left(23^{\circ} \mathrm{C}\right.$ to $25^{\circ} \mathrm{C}$ of temperature and relative humidity of $\sim 60 \%$ ). The athletes were asked to maintain the same routine during the evaluation days, avoiding strenuous exercise and refraining from consuming caffeine for $48-\mathrm{h}$ before the test.

The three types of exercise condition in terms of warm-up were: i) exercise without any previous warm-up; ii) exercise after traditional warm-up (which included dynamic resistance exercises); and iii) exercise after a stretching warm-up (including 3 exercises as 
shown on figure 1). Full explanation of the three types of war-up is described elsewhere $[32,34]$.
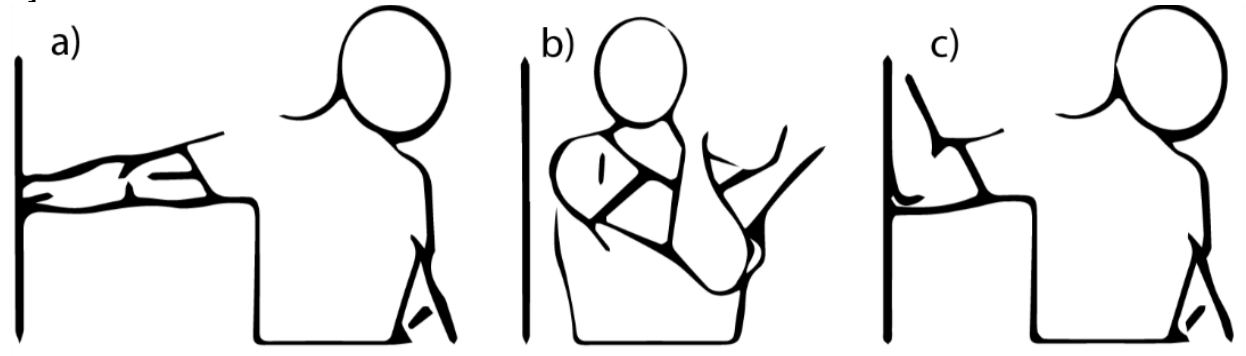

Figure 1. Stretches used as a warm-up method (A) shoulder, (B) triceps and (C) pectoralis major

\subsection{Procedures}

Skin temperature measurement

Thermal images acquisition was performed in a room prepared without natural light, with no airflow directed to the collection site, in ambient temperature conditions maintained around $24^{\circ} \mathrm{C} \pm 1^{\circ} \mathrm{C}$ and relative humidity around $50 \%$ by means of an air conditioner and monitored by a hygrometer (HIGHMED, model HM-01, USA) [11,13].

Subjects were instructed not to perform vigorous physical activity in the previous 24 hours, not to consume alcohol or caffeine, and not to use any type of cream or lotion on the skin in the 6 hours immediately prior to the evaluation. To obtain the thermograms, the athlete remained seated and did not make sudden movements, did not cross the arms, and did not scratch for a period of at least 10 minutes for acclimatization [13,35].

Images were captured by an infrared camera model FLIR T640sc (Flir, Stockholm, Sweden) measuring range $-40{ }^{\circ} \mathrm{C}$ to $2000^{\circ} \mathrm{C}$, accuracy $2 \%$, sensitivity $<0.035$, infrared spectral band of 7.5-14 $\mu \mathrm{m}$, refresh rate of $30 \mathrm{~Hz}$, resolution of $640 \times 480$ pixels. The software used for thermal image analysis was FLIR TOOLS (Flir, Stockholm, Sweden). The region of interest evaluated was the anterior and posterior faces of the trunk and arms [13,36,37]. Figure 2 presents an illustration of the thermal images acquired.

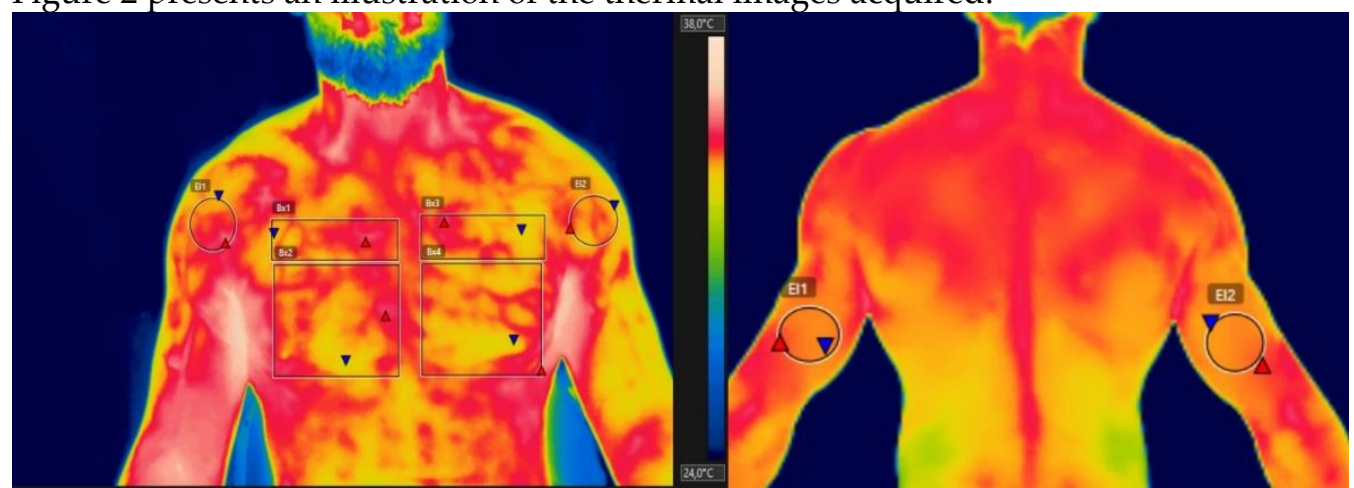

Figure 2. Illustration of the Regions of Interest (ROI) in the thermal images acquired.

Dynamic strength measurements (1-RM and mean propusive velocity)

In every testing herein, a $2.1 \mathrm{~m}$ long official adapted bench (Eleiko Sport AB, Halmstad, Sweden), approved by the International Paralympic Committee [38] was used. The barbell was a $2.2 \mathrm{~m}$ long, $20 \mathrm{~kg}$ weight official bar (Eleiko Sport AB, Halmstad, Sweden). 1- RM assessment in bench press exercise was performed following the protocol proposed by Fleck and Kraemer [39]. A 3- to 5-min rest was provided between attempts. To measure the speed of movement, a valid and reliable [40] linear position transducer (Chronojump, BoscoSystem, Barcelona, Spain) was attached to the bar. The maximum speed averages were collected with the 1-RM load [41]. 
Isometric evaluation was performed by having the subjects pressing the bar at a distance of $15 \mathrm{~cm}$ from the chest. Impulse, variability and peak torque (PT) were measured with the help of a force sensor (Chronojump, BoscoSystem, Barcelona, Spain) and a goniometer FL6010 (Sanny, São José dos Campos, Brazil). Details of this testing can be found elsewhere [32].

\subsection{Statistical Analysis}

After confirmation of normality and homogeneity assumptions, one-way ANOVA with Bonferroni's post hoc was performed to compare the measurements post- the three types of warm up. A repeated-measures analysis of variance was used to evaluate the performance between the warm up conditions, followed by Bonferroni post hoc comparison tests. To check the effect size partial Eta squared $(\eta 2 p)$ was used, adopting values of low effect $(\leq 0.05)$, medium effect $(0.05$ to 0.25$)$, high effect $(0.25$ to 0.50$)$, and very high effect $(>0.50)$ for ANOVA [42]. A d value $<0.2$ was considered a trivial effect, 0.2 to 0.6 a small effect, 0.6 to 1.2 a moderate effect, 1.2 to 2.0 a large effect, 2.0 to 4.0 a very large effect, and $\geq 4.0$ an extremely large effect [43]. Cohen $d$ was calculated as the difference between the mean divided by the pooled SD to estimate the effect size for between-lift comparison [42]. All statistical analyses were performed using the computerized package Statistical Package for the Social Science (SPSS)version 22.0(IBM Corp, Armonk, NY, USA). The level of significance was set at $\mathrm{p}<0.05$. Data is presented as means $(\mathrm{X}) \pm$ standard deviation (SD) and $95 \%$ confidence interval (95\% CI).

\section{Results}

The participants $1-\mathrm{RM}$ in bench press was $119.07 \pm 43.15$, which corresponded to a mean of $1.50 \pm 0.38$ times their body mass. Values above 1.4 (1RM / body weight) in the bench press, are considered to classify elite athletes [44].

The results found in the dynamic indicators of force, MPV and Power, and static, Impulse and Variability in relation to the different types of warm-up are shown in table 1.

Table 1: Dynamic and static force indicators (mean \pm standard deviation, $95 \% \mathrm{CI}$ ) in relation to different types of Warm up.

\begin{tabular}{ccccc}
\hline Warm up & MPV (m.s-1) & Power $(\mathrm{W})$ & Impulse(N.s) & Variability (N) \\
& X \pm DP & X \pm DP & X \pm DP & X \pm DP \\
& $(\mathrm{IC} \mathrm{95 \% )}$ & $(\mathrm{IC} 95 \%)$ & $(\mathrm{IC} 95 \%)$ & $(\mathrm{IC} 95 \%)$ \\
\hline Without Warm & $0,12 \pm 0,06$ & $137,69 \pm 90,80$ & $4022,23 \pm 1341,43$ & $46,74 \pm 30,06$ \\
up & $(0,09-0,16)$ & $(87,41-187,97)$ & $(3279,36-4765,09)$ & $(30,10-63,38)$ \\
Traditional & $0,11 \pm 0,05$ & $128,86 \pm 69,70$ & $3964,91 \pm 1240,10$ & $40,57 \pm 17,72$ \\
& $(0,09-0,14)$ & $(90,26-167,46)$ & $(3278,17-4651,66)$ & $(30,76-50,38)$ \\
Stretching & $0,10 \pm 0,04$ & $105,77 \pm 50,77$ & $3740,41 \pm 1114,96$ & $41,26 \pm 23,42$ \\
& $(0,07-0,12)$ & $(77,65-133,88)$ & $(3122,96-4357,85)$ & $(28,29-54,22)$ \\
P & 0.272 & 0.383 & 0.293 & 0.999 \\
$\eta 2 p$ & $0.122 \# \#$ & $0.116 \# \#$ & $0.121 \# \#$ & $0.030 \#$ \\
\hline
\end{tabular}

* p $<0.05$ (ANOVA two-way, and Bonferroni Post Hoc).\# Low Effect, \#\# Medium Effect MPV: Mean Propulsive Velocity.

Table 3 shows that there were no differences in the dynamic and static indicators of the force in relation to the different types of warm-up. The results found for Peak Torque (Nm) and 1 Maximum Repetition (kg) are shown in Figure 3. 

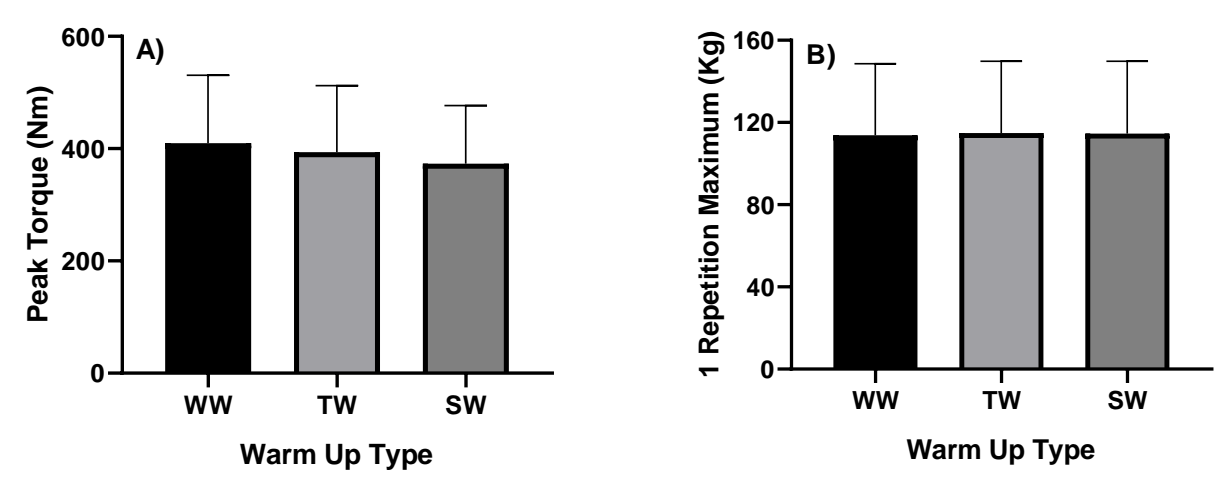

Figure 3. Evaluation of A) Peak Torque (N.m) and B) 1 Repetition Maximum (Kg), with different types of warm up. WW: without warm up, TW: traditional warm up and SW: stretching warm up.

No significant differences were found in relation to the A) Peak Torque ( $p=0.055$, $\mathrm{F}=4.560, \eta 2 \mathrm{p}=0.246$ medium effect $)$, and B) 1 Repetition Maximum ( $\mathrm{p}=0.139, \mathrm{~F}=3.191, \eta 2 \mathrm{p}$ $=0.186$, medium effect) between the different types of warm up.

Table 3. Skin Temperature over active muscles in different types of Warm-Up (Mean \pm SD and CI 95\%).

\begin{tabular}{lcccccccc}
\hline Muscles & $\begin{array}{c}\text { WW } \\
\left({ }^{\circ} \mathrm{C}\right)\end{array}$ & $\begin{array}{c}\text { TW } \\
\left({ }^{\circ} \mathrm{C}\right)\end{array}$ & $\begin{array}{c}\text { SW } \\
\left({ }^{\circ} \mathrm{C}\right)\end{array}$ & $\begin{array}{c}\text { WW vs. } \\
\text { TW }\end{array}$ & $\begin{array}{c}\text { WW vs. } \\
\text { SW }\end{array}$ & $\begin{array}{c}\text { TW vs. } \\
\text { SW }\end{array}$ & p-value & $\eta p 2$ \\
\hline Pectoral & $31,77 \pm 1,18$ & $32,23 \pm 0,80$ & $32,06 \pm 1,09$ & $\mathrm{p}=0.238$ & $\mathrm{p}=0.505$ & $\mathrm{p}=0.642$ & 0.261 & $0.091 \mathrm{a}$ \\
Sternal & $(31,11-32,42)$ & $(31,79-32,68)$ & $(31,45-32,67)$ & $\mathrm{d}=0.456$ & $\mathrm{~d}=0.255$ & $\mathrm{~d}=0.178$ & & \\
Pectoral & $32,51 \pm 0,74^{*}$ & $33,04 \pm 0,71^{*}$ & $33,02 \pm 0,55$ & $\mathrm{p}=0.064$ & $\mathrm{P}=0.049^{*}$ & $\mathrm{p}=0.934$ & $0.038^{*}$ & $0.276 \mathrm{~b}$ \\
Cavicular & $(32,10-32,92)$ & $(32,65-33,43)$ & $(32,72-33,32)$ & $\mathrm{d}=0.731$ & $\mathrm{~d}=0.782$ & $\mathrm{~d}=0.031$ & & \\
Anterior & $32,31 \pm 0,69$ & $32,52 \pm 0,78$ & $32,64 \pm 0,60$ & $\mathrm{p}=0.457$ & $\mathrm{p}=0.230$ & $\mathrm{p}=0.061$ & 0.145 & $0.099 \mathrm{a}$ \\
Deltoide & $(31,93-32,69)$ & $(32,09-32,95)$ & $(32,31-32,97)$ & $\mathrm{d}=0.285$ & $\mathrm{~d}=0.465$ & $\mathrm{~d}=0.741$ & & \\
Triceps & $31,47 \pm 0,93$ & $31,99 \pm 0,54$ & $32,01 \pm 0,80$ & $\mathrm{p}=0.082$ & $\mathrm{p}=0.112$ & $\mathrm{p}=0.939$ & 0.108 & $0.193 \mathrm{a}$ \\
& $(30,96-31,99)$ & $(31,70-32,29)$ & $(31,57-32,46)$ & $\mathrm{d}=0.684$ & $\mathrm{~d}=0.623$ & $\mathrm{~d}=0.029$ & & \\
\hline
\end{tabular}

* $p<0.05$ (ANOVA). (WW in comparison with TW). $\eta p 2$ = partial eta square. "a": medium effect and " $b$ ": high effect.

d: Cohen' d. WW: without warm up, TW: traditional warm up and SW: stretching warm up

\section{Discussion}

The objective of our study was to evaluate the different types of warm-up, without warm-up, traditional warm-up and warm-up with stretching, on thermography and strength indicators. The results of the mean propulsive velocity (MPV) did not show differences between the types of warm-up, however the condition without warm-up (WW) was the one that presented the highest average propulsive speed $(1.12 \pm 0.06 \mathrm{~ms}-1)$, followed by traditional warm-up (TW) $(0.11 \pm 0.05 \mathrm{~ms}-1)$, and warm-up with stretching (SW) $(0.10 \pm 0.04 \mathrm{~ms}-1)$.

A study that evaluated the influence of specific warm-up on strength performance, found that participants were able to achieve a higher propulsive speed in the second and third sets in the squat, and with a tendency to decrease propulsive speed in the bench press, and the time for propulsive speed was shorter after warming up with progressive intensity, demonstrating that speed can be affected by warming up, tending to decrease with more activities [29]. In the horizontal bench press, the MPV does not tend to be greater at the beginning of the training, this is explained by the lower muscle mass involved, when compared to other exercises, in addition to being a relatively simple movement [45]. Thus, it seems that a more relaxed muscle tends to establish a higher speed than after a more traditional warm-up. The reduction in movement speed during strength 
work tends to indicate fatigue $[46,47]$. On the other hand, stretching exercises for sports training [48], as well as for maximum tests or for competition are highly questionable, and would normally be related to loss of performance [49]. On the other hand, the elapsed time of the warm-up with the use of stretching must observe an interval greater than three minutes and the warm-up continues [34].

The power despite not showing differences between the three types of warm-up, the condition without warm-up showed a higher power $(137.69 \pm 90.80 \mathrm{~W})$, followed by traditional warm-up $(128.86 \pm 69.70 \mathrm{~W})$, and warm-up with stretching $(105,77 \pm 50.77 \mathrm{~W})$. If we consider that power is the product of strength by speed, the importance of speed in resistance training, and can be considered very important in the assessment of muscle strength $[50,51]$. The warm-up, whether traditional or even with pre-activation, aims at increasing the muscle temperature, activation of the motor unit and myofiber water content $[52,53]$. Moderate to heavy exercises with loads varying between $20-90 \%$ of a maximum repetition tend to improve sprint and jump, especially in subjects trained and familiar with the exercise load [54,55]. However, our findings indicate that WW and TW tend to be better than SW. On the other hand, contrary to this, dynamic and static stretching tends to be favorable as a warm-up strategy. In active individuals, dynamic stretching increased the height of the vertical jump. On the other hand, agility tends to be positively impacted by stretching. Dynamic stretching can improve an athlete's power [56].

The same kinetics occurred in relation to the static components of the force, where there were no differences in Impulse, and the WW method obtained the highest value $(4022.23 \pm 1341.43 \mathrm{Ns})$, followed by TW $(3964.91 \pm 1240.10 \mathrm{Ns})$ and of the SW (3740.41 \pm $1114.96 \mathrm{Ns})$. In the same direction, the participants tested after 6 min of swimming warm up or warm up on land, with 3 repetitions (pull-over at $85 \%$ of the maximum of one repetition). Speed, force, acceleration, impulse, rate of force development (RFD) were evaluated. Warm-up on land with higher loads increased RFD $(34.52 \pm 16.55$ vs. $31.29 \pm 13.70 \mathrm{~N}$ / s; $\Delta=9.35 \%)$ and stroke rate $(64.70 \pm 9,84 \mathrm{vs} .61 .56 \pm 7.07 \mathrm{~Hz} ; \Delta=5.10 \%)$ compared to traditional water warm-up, but decreased speed, strength, acceleration, impulse and power [57]. That is, traditional warm-up can decrease the impulse, and lighter warm-up tends not to decrease the impulse. Likewise, exercises with high load resistance have been used to facilitate the improvement of neuromuscular performance. Traditional warm-up has its use restricted, despite its specificity and practicality for sports performance. Thus, when verifying the effect of repeated exercises on performance, where 43 subjects were evaluated. Performance was quantified through vertical jump, relative thrust, and normalized peak strength at baseline. No improvements were found for the relative impulse in repeated trials, the sixth trial was significantly less than the baseline $(2.35 \pm 0.38 \mathrm{vs}$. 2.26 $\pm 0.35 ; \mathrm{p} \leq 0.001)$. This indicates that the repetition of traditional warm-ups can lead to fatigue, which tends to interfere with performance [58].

In variability, although there are no differences between the types of warm-up, the least variability was TW $(40.57 \pm 17.72 \mathrm{~N})$, followed by SW $(41.26 \pm 23.42 \mathrm{~N})$ and WW $(46$, $74 \pm 30.06 \mathrm{~N})$. This may indicate that traditional warm-up tends to promote a more stable situation on a muscular level than other types of warm-up, noting however that there were no significant differences between the warm-up methods.

There were also no differences in Peak Torque, however the WW method showed higher values $(409.58 \pm 120.99 \mathrm{Nm})$, followed by TW $(393.74 \pm 118.48 \mathrm{Nm})$ and SW $(373.14$ $\pm 103.51 \mathrm{Nm}$ ). One study evaluated standard warming or dropjump (plyometric protocol) or a slow walk (control protocol). Post-activation potentiation was assessed by changes in isometric muscle contractions. The plyometric protocol increased the peak contraction torque (PTT), the rate of torque development (RTD) and the impulse significantly (by 23, 39 and $46 \%$, respectively). Peak contraction torque, RTD and impulse decreased significantly after standard warm-up. Thus, standard warming did not enhance, but may have reduced, the muscle's ability to generate strength [59]. The data in this study contradict our findings.

In the $1 \mathrm{RM}$ test, the three methods also showed no differences, however the TW method $(114.80 \pm 34.98 \mathrm{~kg})$, showed higher values, followed by the SW $(114.53 \pm 35.20 \mathrm{~kg})$ 
and the WW $(113.80 \pm 34.80 \mathrm{~kg})$. A specific warm-up can increase the production of strength after maximum or almost maximum muscle stimulation [31]. The effects of warming on athletic success have gained great attention in recent studies. Authors [32] evaluated different types of warm-up, with the participation of 15 elite Brazilian male athletes from Paralympic Powerlifting (age, $24.14 \pm 6.21$ years; body weight, $81.67 \pm 17.36$ $\mathrm{kg}$ ). A significant difference was observed for the maximum isometric strength, in the without warm-up (WW) in relation to traditional warm-up (TW) and stretching warm-up (SW) $(p=0.005, \eta 2 p=0.454$, high effect). On the other side, no significant differences were observed in the RFD, fatigue index (FI), and time in the different types of warm-up ( $p>$ $0.05)$. No significant differences were observed in relation to the maximum repetition $(p=$ $0.121, \eta 2 p=0.275$, medium effect) or the maximum speed $(p=0.712, \eta 2 p=0.033$, low effect) between the different types of warm-up. The different warm-up methods do not seem to provide significant differences in strength indicators in this population, and this could be explained by the displacement they use to the upper limbs, the target of the study [32].

In thermographic analysis, there was significant difference only in the Pectoral muscle clavicular portion between the TW $\left(33.04 \pm 0.71^{\circ} \mathrm{C}\right)$ and the $\mathrm{WW}\left(32.51 \pm 0.74^{\circ} \mathrm{C}\right)(\mathrm{p}=$ 0.038). The TW method also presented slightly higher values than the SW and WW in Pectoral Muscles Sternal portion and in Deltoid anterior portion, but with $\mathrm{p}$ value $>0.05$. These results are in agreement with authors who studied thermal response to resistance training [60,61]; since traditional warm-up involves specific resistance exercises for the primary muscles that are recruited in the main work.

The physiological reason for the increase in skin temperatures observed in the Pectoral muscle clavicular portion (TW protocol) might be the increase in the recruitment of motor units, which occurs during the traditional warm-up, needed to prepare the muscles for the considerable effort needed to overcome the weight of the barbell and give it acceleration [62].

Authors $[60,61]$ reported an increase in skin temperature over the muscles that were the main responsible for the movement requested, after the exercise. Neves et al., [37] report that the warming of ROI in arm exercise seems to be related to exercise volume. In this sense, since the traditional warm-up protocol included a large volume of exercises, it promoted an increase in blood flow to the Pectoral muscle clavicular portion and, consequently, greater heat dissipation by the skin over this muscle.

The study used the functional classification adopted by the International Paralympic Committee. Thus, it can be mentioned as limitations of this study the control of variables such as balance, food, and life habits.

\section{Conclusions}

It can be concluded that the type of warm-up does not seem to interfere with the performance of Paralympic Powerlifting athletes. However, although there are no significant differences between the warm-up methods, the thermal images showed that traditional warm-up best meets the objectives expected for this preparation phase and that in a competition it could be enough to provide better performance and classification.

The results found may have been influenced by the condition of being in a wheelchair and requiring the use of the muscles of the upper limbs and trunk for displacement, thus promoting the maintenance of these muscle groups in a state of activity similar to what is observed after the warm-up protocols.

Author Contributions: For research articles with several authors, a short paragraph specifying their individual contributions must be provided. The following statements should be used "Conceptualization, X.X. and Y.Y.; methodology, X.X.; software, X.X.; validation, X.X., Y.Y. and Z.Z.; formal analysis, X.X.; investigation, X.X.; resources, X.X.; data curation, $X . X . ;$ writing - original draft preparation, $X . X . ;$ writing - review and editing, $X . X . ;$ visualization, $X . X . ;$ supervision, $X . X . ;$ project administration, X.X.; funding acquisition, Y.Y. All authors have read and agreed to the published 
version of the manuscript." Please turn to the CRediT taxonomy for the term explanation. Authorship must be limited to those who have contributed substantially to the work reported.

Funding: "This research received no external funding"

Institutional Review Board Statement: In this section, please add the Institutional Review Board Statement and approval number for studies involving humans or animals. Please note that the Editorial Office might ask you for further information. Please add "The study was conducted according to the guidelines of the Declaration of Helsinki, and approved by the Institutional Review Board (or Ethics Committee) of NAME OF INSTITUTE (protocol code XXX and date of approval)." OR “Ethical review and approval were waived for this study, due to REASON (please provide a detailed justification)." OR “Not applicable." for studies not involving humans or animals. You might also choose to exclude this statement if the study did not involve humans or animals.

Informed Consent Statement: Any research article describing a study involving humans should contain this statement. Please add "Informed consent was obtained from all subjects involved in the study." OR "Patient consent was waived due to REASON (please provide a detailed justification)." OR "Not applicable." for studies not involving humans. You might also choose to exclude this statement if the study did not involve humans.

Written informed consent for publication must be obtained from participating patients who can be identified (including by the patients themselves). Please state "Written informed consent has been obtained from the patient(s) to publish this paper" if applicable.

Data Availability Statement: In this section, please provide details regarding where data supporting reported results can be found, including links to publicly archived datasets analyzed or generated during the study. Please refer to suggested Data Availability Statements in section "MDPI Research Data Policies" at https://www.mdpi.com/ethics. You might choose to exclude this statement if the study did not report any data.

Acknowledgments: In this section, you can acknowledge any support given which is not covered by the author contribution or funding sections. This may include administrative and technical support, or donations in kind (e.g., materials used for experiments).

Conflicts of Interest: “The authors declare no conflict of interest."

\section{References}

1. Moreira OC, Faraci LL, de Matos DG, Mazini Filho ML, da Silva SF, Aidar FJ, Hickner RC, de Oliveira CE. Cardiovascular Responses to Unilateral, Bilateral, and Alternating Limb Resistance Exercise Performed Using Different Body Segments.J Strength Cond Res. 2017 Mar;31(3):644-652. doi: 10.1519/JSC.0000000000001160.

2. Costa Pereira LM, Aidar FJ, de Matos DG, de Farias Neto JP, de Souza RF, Sobral Sousa AC, de Almeida RR, Prado Nunes MA, Nunes-Silva A, da Silva Júnior WM. Assessment of Cardiometabolic Risk Factors, Physical Activity Levels, and Quality of Life in Stratified Groups up to 10 Years after Bariatric Surgery.Int J Environ Res Public Health. 2019 Jun 4;16(11):1975. doi: 10.3390/ijerph16111975.

3. Pereira L, Batista Oliveira V, Menezes Oliveira JL, Soares Barreto-Filho JA, Almeida-Santos MA, de Souza RF, de Freitas Zanona A, Machado Reis V, Aidar FJ, Sobral Sousa AC. A Retrospective Study about the Differences in Cardiometabolic Risk Indicators and Level of Physical Activity in Bariatric Surgery Patients from Private vs. Public Units. Rocha de Almeida R, Cândido de Souza MF, Gama de Matos D, Monteiro Costa Int J Environ Res Public Health. 2019 Nov 27;16(23):4751. doi: 10.3390/ijerph16234751.

4. Aidar FJ, Carneiro AL, Costa Moreira O, Patrocínio de Oliveira CE, Garrido ND, Machado Reis V, Raineh I, Vilaça JM, Gama de Matos D. Effects of resistance training on the physical condition of people with multiple sclerosis. J Sports Med Phys Fitness. 2018 Jul-Aug;58(7-8):1127-1134. doi: 10.23736/S0022-4707.17.07621-6.

5. Aidar FJ, de Oliveira RJ, de Matos DG, Mazini Filho ML, Moreira OC, de Oliveira CE, Hickner RC, Reis VM. A Randomized Trial Investigating the Influence of Strength Training on Quality of Life in Ischemic Stroke Top Stroke Rehabil. 2016 Apr;23(2):84-9. doi: 10.1080/10749357.2015.1110307.

6. Aidar FJ, Gama de Matos D, de Souza RF, Gomes AB, Saavedra F, Garrido N, Carneiro AL, Reis V. Influence of aquatic exercises in physical condition in patients with multiple sclerosis.J Sports Med Phys Fitness. 2018 May;58(5):684-689. doi: 10.23736/S0022-4707.17.07151-1.

7. Aidar FJ, Jacó de Oliveira R, Gama de Matos D, Chilibeck PD, de Souza RF, Carneiro AL, Machado Reis V. A randomized trial of the effects of an aquatic exercise program on depression, anxiety levels, and functional capacity of people who suffered an ischemic stroke. J Sports Med Phys Fitness. 2018 Jul-Aug;58(7-8):1171-1177. doi: 10.23736/S0022-4707.17.07284$\mathrm{X}$. 
8. Matos DG, Mazini Filho ML, Moreira OC, DE Oliveira CE, DE Oliveira Venturini GR, DA Silva-Grigoletto ME, Aidar FJ. Effects of eight weeks of functional training in the functional autonomy of elderly women: a pilot study.J Sports Med Phys Fitness. 2017 Mar;57(3):272-277. doi: 10.23736/S0022-4707.16.06514-2.

9. Mazini Filho ML, Aidar FJ, Gama de Matos D, Costa Moreira O, Patrocínio de Oliveira CE, Rezende de Oliveira Venturini G, Magalhães Curty V, Menezes Touguinha H, Caputo Ferreira ME. Circuit strength training improves muscle strength, functional performance and anthropometric indicators in sedentary elderly women. J Sports Med Phys Fitness. 2018 JulAug;58(7-8):1029-1036. doi: 10.23736/S0022-4707.17.06903-1.

10. Moreira OC, Lopes GS, de Matos DG, Mazini-Filho ML, Aidar FJ, Silva SF, de Oliveira CE. Impact of two hydrogymnastics class methodologies on the functional capacity and flexibility of elderly women. J Sports Med Phys Fitness. 2019 Jan;59(1):126-131. doi: 10.23736/S0022-4707.17.07872-0.

11. Zanona AF, de Souza RF, Aidar FJ, de Matos DG, Santos KMB, Paixão MDC, Sampaio PYS, Almeida Junior H, MonteSilva KK. Use of Virtual Rehabilitation to Improve the Symmetry of Body Temperature, Balance, and Functionality of Patients with Stroke Sequelae. Ann Neurosci. 2019 Jan;25(3):166-173. doi: 10.1159/000488581.

12. Paz ÂA, Aidar FJ, de Matos DG, de Souza RF, da Silva-Grigoletto ME, van denTillaar R, Ramirez-Campillo R, Nakamura FY, Costa MDC, Nunes-Silva A, Costa E Silva AA, Marçal AC, Reis VM. Comparison of Post-Exercise Hypotension Responses in Paralympic Powerlifting Athletes after Completing Two Bench Press Training Intensities. Medicina (Kaunas). 2020 Apr 1;56(4):156. doi: 10.3390/medicina56040156.

13. Almeida Barros N, Aidar FJ, DE Matos DG, DE Souza RF, Neves EB, DE Araujo Tinoco Cabral BG, Carmargo EA, Reis VM. Evaluation of Muscle Damage, Body Temperature, Peak Torque, and Fatigue Index in Three Different Methods of Strength Gain. Int J Exerc Sci. 2020 Sep 1;13(3):1352-1365.

14. Rodrigues JA, Pereira ET, Lopes JS, da Fonseca Silva MV, Resende NM, Fernandes da Silva S, Aidar FJ, Patrocinio de Oliveira CE, Costa Moreira O. Effects of circuit resistance training on muscle power, functional agility, and bones' mineral content in people with spinal injury.J Sports Med Phys Fitness. 2020 (epubahead). doi: 10.23736/S0022-4707.20.11286-6.

15. Rosa Santos LD, Araujo SS, Vieira EFDS, Estevam CDS, Santos JLD, Wichi RB, Lima FB, Carvalho CRO, Aidar FJ, Marçal AC. Effects of 12 Weeks of Resistance Training on Cardiovascular Risk Factors in School Adolescents.Medicina (Kaunas). 2020 May 6;56(5):220. doi: 10.3390/medicina56050220.

16. Santos MDM, Aidar FJ, de Souza RF, Dos Santos JL, da Silva de Mello A, Neiva HP, Marinho DA, Marques MC. Does the Grip Width Affect the Bench Press Performance of Paralympic Powerlifters? Int J Sports Physiol Perform. 2020 Sep 11:1-8. doi: 10.1123/ijspp.2019-0784.

17. Szafraniec R, Kisilewicz A, Kumorek M, Kristiansen M, Madeleine P, Mroczek D. Effects of High-Velocity Strength Training on Movement Velocity and Strength Endurance in Experienced Powerlifters with Cerebral Palsy. J Hum Kinet. 2020 Jul 21;73:235-243. doi: 10.2478/hukin-2020-0009

18. Fraga GS, Aidar FJ, Matos DG, Marçal AC, Santos JL, Souza RF, Carneiro AL, Vasconcelos AB, Da Silva-Grigoletto ME, van den Tillaar R, Cabral BT, Reis VM. Effects of Ibuprofen Intake in Muscle Damage, Body Temperature and Muscle Power in Paralympic Powerlifting Athletes. Int J Environ Res Public Health. 2020 Jul 17;17(14):5157. doi: 10.3390/ijerph17145157.

19. Gołaś A, Zwierzchowska A, Maszczyk A, Wilk M, Stastny P, Zając A. Neuromuscular Control During the Bench Press Movement in an Elite Disabled and Able-Bodied Athlete. J Hum Kinet. 2017 Dec 28;60:209-215. doi: 10.1515/hukin-20170110.

20. Willick SE, Cushman DM, Blauwet CA, Emery C, Webborn N, Derman W, Schwellnus M, Stomphorst J, Van de Vliet P. The epidemiology of injuries in powerlifting at the London 2012 Paralympic Games: An analysis of 1411 athletedays.Scand J Med Sci Sports. 2016 Oct;26(10):1233-8. doi: 10.1111/sms.12554.

21. Ribeiro Neto F, Dorneles JR, Luna RM, Spina MA, Gonçalves CW, Gomes Costa RR. Performance Differences Between the Arched and Flat Bench Press in Beginner and Experienced Paralympic Powerlifters.J StrengthCond Res. 2020 Jul 27. doi: 10.1519/JSC.0000000000003736.

22. Ribeiro Neto F, Dorneles JR, Costa RRG. Are the flat and arched bench press really similar? Sports Biomech. 2020 Apr 22:1-2. doi: 10.1080/14763141.2020.1751872

23. Soares Freitas Sampaio CR, Aidar FJ, Ferreira ARP, Santos JLD, Marçal AC, Matos DG, Souza RF, Moreira OC, Guerra I, Fernandes Filho J, Marcucci-Barbosa LS, Nunes-Silva A, Almeida-Neto PF, Cabral BGAT, Reis VM. Can Creatine Supplementation Interfere with Muscle Strength and Fatigue in Brazilian National Level Paralympic Powerlifting? Nutrients. 2020 Aug 19;12(9):E2492. doi: 10.3390/nu12092492.

24. McGowan CJ, Pyne DB, Thompson KG, Rattray B. Warm-Up Strategies for Sport and Exercise: Mechanisms and Applications. Sports Med. 2015 Nov; 45(11):1523-46. doi: 10.1007/s40279-015-0376-x.

25. Bishop, D. Warm up I: Potential mechanisms and the effects of passive warm up on exercise performance. Sports Med. 2003, 33, 439-454. [Google Scholar] [CrossRef] [PubMed]

26. Bishop, D. Warm up II: Performance changes following active warm up and how to structure the warm up. Sports Med. 2003, 33, 483-498. [Google Scholar] [CrossRef] [PubMed]

27. Gil MH, Neiva HP, Garrido ND, Aidar FJ, Cirilo-Sousa MS, Marques MC, Marinho DA. The Effect of Ballistic Exercise as Pre-Activation for 100 m Sprints.Int J Environ Res Public Health. 2019 May 24;16(10):1850. doi: 10.3390/ijerph16101850.

28. Gil, M.H.; Neiva, H.P.; Sousa, A.C.; Marques, M.C.; Marinho, D.A. Current approaches on warming up for sports performance: A critical review. Strength Cond. J. 2019, 41, 70-79. [Google Scholar] [CrossRef] 
29. Ribeiro B, Pereira A, Neves PP, Sousa AC, Ferraz R, Marques MC, Marinho DA, Neiva HP. The Role of Specific Warm-up during Bench Press and Squat Exercises: A Novel Approach. Int J Environ Res Public Health. 2020 Sep 22;17(18):E6882. doi: 10.3390/ijerph17186882

30. Neiva, H.P.; Marques, M.C.; Barbosa, T.M.; Izquierdo, M.; Viana, J.L.; Teixeira, A.; Marinho, D.A. Warm-up volume affects the $100 \mathrm{~m}$ swimming performance: A randomized crossover study. J. Strength Cond. Res. 2015, 29, 3026-3036. [Google Scholar] [CrossRef]

31. Junior, D.; Junor, A.; Serpa, É.; Gomes, W.; Soares, E.; Lopes, C.; Teixeira, L.; Marchetti, P. Different warm-ups on the maximum repetition performance in resistance training. Rev. Bras. Med. Esporte 2014, 20, 461-464. [Google Scholar]

32. Resende MA, Vasconcelos Resende RB, Reis GC, Barros LO, Silva Bezerra MR, Matos DG, Marçal AC, Almeida-Neto PF, Cabral BGAT, Neiva HP, Marinho DA, Marques MC, Reis VM, Garrido ND, Aidar FJ. The Influence of Warm-Up on Body Temperature and Strength Performance in Brazilian National-Level Paralympic Powerlifting Athletes. Medicina 2020, 56(10), 538; https://doi.org/10.3390/medicina56100538

33. Adelsberger, R.; Tröster, G. Effects of stretching and warm-up routines on stability and balance during weight-lifting: A pilot investigation. BMC Res. 2014, 7, 938. [CrossRef]

34. De Souza, R.F.; de Matos, D.G.; Nogueira, A.C.; Ferreira, A.R.P.; de Freitas Zanona, A.; Aidar FJ. Analysis of muscle recovery time after acute stretching at peak torque of the hamstring muscles. Med. Dello Sport 2019, 72, 171-180.

35. Marins J, Fernández-Cuevas I, Arnaiz-Lastras J, Sillero-Quintana M. Applications of Infrared Thermography in Sports. A Review. Revista Inter Med Ciencias Activ Física Depor 15: 805-824, 2015

36. Salamunes A, Stadnik A, Neves E. The effect of body fat percentage and body fat distribution on skin surface temperature with infrared thermography. J ThermBiol 66: 1-9, 2017.

37. Neves EB, Salamunes ACC, de Oliveira RM, Stadnik AMW. Effect of body fat and gender on body temperature distribution.J Therm Biol. 2017;70:1-8.

38. International Paralympic Comitê (IPC). Sports. Available online: https://www.paralympic.org/powerlifting(accessed on 10 January 2020).

39. Fleck, S.J.; Kraemer, W.J. Designing Resistance Training Programs, 4th ed.; Human Kinetics: Champaign, IL,USA, 2004.

40. Pérez-Castilla, A.; Piepoli, A.; Delgado-García, G.; Garrido-Blanca, G.; García-Ramos, A. Reliability andconcurrent validity of seven commercially available devices for the assessment of movement velocity atdifferent intensities during the bench press. J. Strength Cond. Res. 2019, 33, 1258-1265. [CrossRef]

41. García-Ramos, A.; Ha_, G.G.; Padial, P.; Feriche, B. Reliability of power and velocity variables collectedduring the traditional and ballistic bench press exercise. Sports Biomech. 2018, 17, 117-130. [CrossRef]

42. Cohen J. Statistics a power primer. Psychol Bull. 1992;112:155-159. PubMed ID: 19565683 doi:10.1037/0033-2909.112.1.155

43. Hopkins WG, Marshall SW, Batterham AM, Hanin J. Progressive statistics for studies in sports medicine and exercise science. Med Sci Sports Exerc. 2009;41(1):3-13. PubMed ID: 19092709 doi:10.1249/MSS.0b013e31818cb278

44. Ball, R.; Weidman, D. Analysis of USA powerlifting federation data from January 1, 2012-June 11, 2016.J. Strength Cond. Res. 2018, 32, 1843-1851. [CrossRef]

45. Evans, A.K.; Durham, M.P.; Hodgkins, T.D.; Sinclair, D.R.; Adams, K.J. Acute effect of bench press on power output during a subsequent ballistic bench throw. Med. Sci Sports, 2001, 33, 325. [Google Scholar] [CrossRef]

46. Pareja-Blanco, F.; Rodríguez-Rosell, D.; Sánchez-Medina, L.; Sanchis-Moysi, J.; Dorado, C.; Mora-Custodio, R.; YáñezGarcía, J.M.; Morales-Alamo, D.; Pérez-Suárez, I.; Calbet, J.; et al. Effects of velocity loss during resistance training on athletic performance, strength gains and muscle adaptations. Scand J. Med. Sci Sports 2017, 27, 724-735. [Google Scholar] [CrossRef] [PubMed]

47. Sanchez-Medina, L; González-Badillo, JJ Perda de velocidade como um indicador de fadiga neuromuscular durante o treinamento de resistência. Med. Sci. Sport Exer. 2011, 43, 1725-1734. [Google Scholar] [CrossRef] [PubMed]

48. Behm DG, Blazevich AJ, Kay AD, McHugh M. Acute Effects of Muscle Stretching on Physical Performance, ROM and Injury Incidence in Healthy Active Individuals: A Systematic Review. Appl Physiol Nutr Metab. 2015;41(1):1-11.

49. Kallerud H, Gleeson N. Effects of stretching on performances involving stretch-shortening cycles. Sports Medicine. 2013;43(8):733-50.

50. González-Badillo, J.J.; Marques, M.C.; Sánchez-Medina, L. The importance of movement velocity as a measure to control resistance training intensity. J. Hum. Kinet.2011, 29, 15-19. [Google Scholar] [CrossRef]

51. González-Badillo, J.J.; Sánchez-Medina, L. Movement velocity as a measure of loading intensity in resistance training. Int. J. Sports Med.2010,31, 347-352. [Google Scholar]

52. Blazevich AJ, Babault N. Pós-ativação potenciação cersus pós-ativação aprimoramento de desempenho em humanos: perspectiva histórica, mecanismos subjacentes e questões atuais. Frente. Physiol. 2019; 10: 1359. doi: 10.3389 / fphys.2019.01359. [Artigogratuito PMC] [PubMed] [ CrossRef ] [ Google Scholar ]

53. Tomlinson KA, Hansen K, Helzer D, Lewis ZH, Leyva WD, McCauley M, Pritchard W, Silvestri E, Quila M, Yi M, Jo E. The Effects of Loaded Plyometric Exercise during Warm-Up on Subsequent Sprint Performance in Collegiate Track Athletes: A Randomized Trial. Sports (Basel). 2020 Jul 17;8(7):101. doi: 10.3390/sports8070101.

54. Jo E., Judelson D.A., Brown L.E., Coburn J.W., Dabbs N.C. Influence of recovery duration after a potentiating stimulus on muscular power in recreationally trained individuals. J. Strength. Cond. Res. 2010;24:343-347. doi: 10.1519/JSC.0b013e3181cc22a4. [PubMed] [CrossRef] [Google Scholar] 
55. Duncan M.J., Thurgood G., Oxford S.W. Effect of heavy back squats on repeated sprint performance in trained men. J. Sports Med. Phys. Fit. 2014; 54:238-243. [PubMed] [Google Scholar]

56. Anderson BL, Harter RA, Farnsworth JL. The Acute Effects of Foam Rolling and Dynamic Stretching on Athletic Performance: A Critically Appraised Topic. J Sport Rehabil. 2020 Aug 13:1-6. doi: 10.1123/jsr.2020-0059

57. Cuenca-Fernández F, Batalha NM, Ruiz-Navarro JJ, Morales-Ortiz E, López-Contreras G, Arellano RPost high intensity pull-over semi-tethered swimming potentiation in national competitive swimmers. .J Sports Med Phys Fitness. 2020 Jun 30. doi: 10.23736/S0022-4707.20.11136-8.

58. Horan SA, Watson SL, Lambert C, Weeks BK. Lunging. Exercise Potentiates a Transient Improvement in Neuromuscular Performance in Young Adults. J Strength Cond Res. 2015 Sep;29(9):2532-7. doi: 10.1519/JSC.0000000000000913.

59. Johnson M, Baudin P, Ley AL, Collins DF. A Warm-Up Routine That Incorporates a Plyometric Protocol Potentiates the Force-Generating Capacity of the Quadriceps Muscles. J Strength Cond Res. 2019 Feb;33(2):380-389. doi: 10.1519/JSC.0000000000002054.

60. Uchôa, P., Matos, F., Neves, E. B., Saavedra, F., Rosa, C., Reis, V. M., \& Vilaça-Alves, J. (2018). Evaluation of two different resistance training volumes on the skin surface temperature of the elbow flexors assessed by thermography. Infrared Physics \& Technology, 93, 178-183.

61. Neves, E. B., Moreira, T. R., Lemos, R., Vilaça-Alves, J., Rosa, C., \& Reis, V. M. (2015). Using skin temperature and muscle thickness to assess muscle response to strength training. Revista Brasileira de Medicina do Esporte, 21(5), 350-354.

62. Kuniszyk-Jóźkowiak, W., Jaszczuk, J., Czaplicki, A., \& Szyszka, P. (2019). Variability of shoulder girdle temperature in the initial phase of the snatch in weightlifting. Acta of Bioengineering and Biomechanics, 21(3):143-148. DOI: 10.5277/ABB01423-2019-02 